\title{
Pulse compression with supercontinuum generation in microstructure fibers
}

\author{
Birgit Schenkel, Rüdiger Paschotta, and Ursula Keller \\ Department of Physics, Institute of Quantum Electronics, Swiss Federal Institute of Technology (ETH), \\ ETH Zürich Hönggerberg, Wolfgang-Pauli-Str. 16, 8093 Zürich, Switzerland
}

Received June 30, 2004; revised manuscript received November 1, 2004; accepted November 5, 2004

\begin{abstract}
We demonstrate the generation of 5.5-fs pulses by dispersive compression of a supercontinuum generated with 15-fs pulses from a Ti:sapphire laser in a 5-mm-long microstructure fiber. The generated continuum is characterized with a setup for cross-correlation spectral-phase interferometry for direct electric-field reconstruction (SPIDER). The reconstructed spectral phase is used as on input for an iterative algorithm optimizing the compressor phase profile. We also discuss the limitations of this technique concerning the achievable pulse duration, including aspects like limited spectral coherence of the supercontinuum, limited compressor resolution, and a limitation of the SPIDER technique. The coherence is limited by power fluctuations and the instability of the seed laser. (C) 2005 Optical Society of America
\end{abstract}

OCIS codes: $320.5520,320.5540,320.0320,190.4370$.

\section{INTRODUCTION}

Microstructure fibers ${ }^{1}$ represent a new kind of fiber consisting of a solid core surrounded by an array of air holes. These fibers have already found applications in many fields, one of the most important applications being spectral broadening. Compression of pulses with high average powers of up to $18 \mathrm{~W}$ has been demonstrated by use of a large-mode-area fiber. ${ }^{2}$ Small-mode-area fibers are used for supercontinuum generation, where not only does the small mode area result in a high peak intensity but also the modified dispersion characteristics of these fibers play an important role.

With these fibers supercontinuum generation has become possible even with nanojoule pulses directly generated by a mode-locked Ti:sapphire laser. ${ }^{3}$ The continua generated in these fibers show some remarkable properties: They can span an entire optical octave or more, are spatially coherent, and have a brightness which exceeds the brightness of a light bulb by at least 5 orders of magnitude. These supercontinua have already found applications in optical coherence tomography ${ }^{4}$ and frequency metrology, where they deliver precise octave-spanning frequency combs. ${ }^{5,6}$ The broad achievable bandwidth also suggests that with appropriate dispersive compression, such supercontinua should allow the generation of very short femtosecond pulses in the few-cycles regime. However, the shortest pulse duration achieved with this approach was $25 \mathrm{fs}^{7,8}$ until very recently, when 6.6-fs pulses were obtained. ${ }^{9}$ Here we demonstrate successful compression down to only $5.5 \mathrm{fs}$ and also discuss in depth the limitations of this technique.

It is known ${ }^{10}$ that the spectral coherence of supercontinua generated in strongly nonlinear processes can be imperfect, and that this can inhibit the compression process, because the dispersive compressor can be adjusted only relatively slowly and thus cannot be optimized for each pulse separately when the spectral phase undergoes strong fluctuations. Particularly for very broad spectra, this problem can be severe. However, it is also strongly affected by the detailed parameters of the fiber (in particular, by its dispersion) and of the initial pulses. In Section 4, we investigate this issue for our fibers using numerical simulations of the pulse propagation. Also, we discuss effects of the limited compressor resolution and a limitation of the spectral-phase interferometry for direct electric-field reconstruction (SPIDER) technique, which has been shown to be suitable for the characterization of pulses with durations below $10 \mathrm{fs} .{ }^{11}$ These issues have been discussed only partially in previous articles. Finally, we arrive at some conclusions on the overall potential of this compression technique.

An alternative method for supercontinuum generation is based on propagation in hollow fibers. ${ }^{12}$ Compared with microstructure fibers, much higher pulse energies in the order of $0.1-1 \mathrm{~mJ}$ are required for strong spectral broadening in hollow fibers. This makes it necessary to use amplified pulses from an amplifier, operating at a repetition rate of, e.g., $1 \mathrm{kHz}$. Of course, the resulting pulse energies are correspondingly higher, making it easier to do a precise SPIDER characterization. The spectral phase of the output of a hollow fiber was investigated with a single-shot SPIDER technique and was found to be very stable $\mathrm{e}^{13}$; this is attributed to self-phase modulation being the dominant broadening mechanism, not significantly deteriorating the spectral coherence. Dispersive compression of such pulses with a similar pulse shaper has enabled the generation of pulses with durations of 3.8 fs (Ref. 14), 3.4 fs (Ref. 15), and even 2.8 fs (Ref. 16).

Compared with hollow-fiber compression, the compression of microstructure fiber supercontinua has the advantage of being possible with much lower pulse energies (well below $100 \mathrm{~nJ}$ ) and therefore at the full laser repetition rate.

\section{EXPERIMENTAL SETUP}

\section{A. Supercontinuum Generation}

Figure 1 shows our experimental setup. The seed laser is a Kerr lens mode-locked Ti:sapphire laser where disper- 


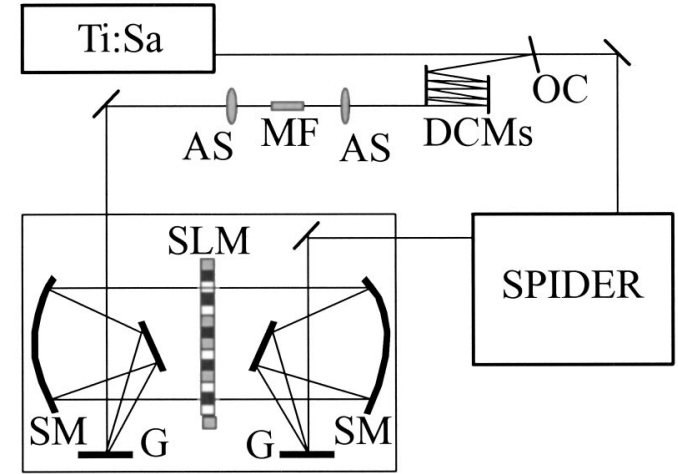

Fig. 1. Experimental setup. Ti:Sa, Ti:sapphire oscillator; OC, output coupler; DCMs, double-chirped mirrors; AS, aspheric lens; MF, microstructure fiber; SM, spherical mirror; G, grating, and SLM, spatial light modulator.

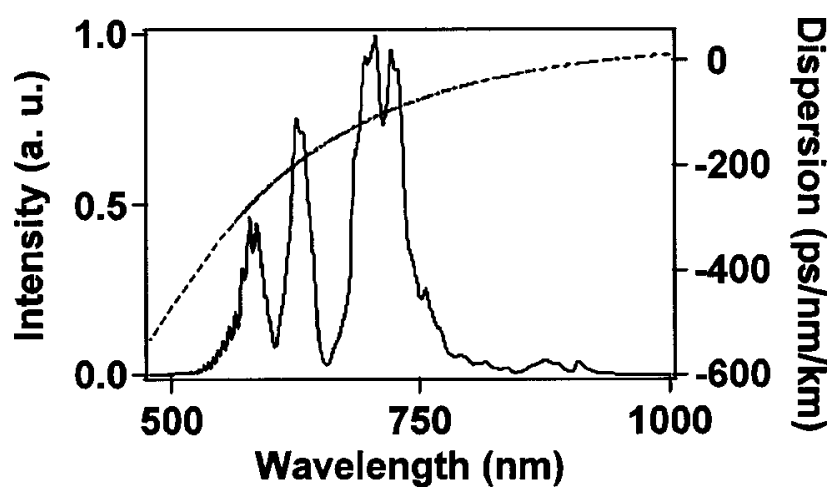

Fig. 2. Solid curve, experimentally measured output spectrum of the 2.6- $\mu \mathrm{m}$-core-diameter fiber; dotted curve, the dispersion profile of this fiber.

sion compensation is achieved with a combination of seven double-chirped mirrors ${ }^{17}$ and a prism pair. ${ }^{18}$ The laser generates 15 -fs pulses with a pulse energy of $16 \mathrm{~nJ}$, a center wavelength of $790 \mathrm{~nm}$, and a repetition rate of 19 $\mathrm{MHz}$. The relatively low repetition rate was chosen in order to obtain output pulses with a higher pulse energy, as required for achieving broad spectra in very short pieces (a few millimeters) of microstructure fiber.

We have chosen to work with such short fiber pieces because we need to limit the required compressor resolution; the dispersive compressor can control the spectral phase for only a discrete set of frequency components. The transmission phase should not vary by more than $\approx 1$ rad between adjacent spectral components. Thus the achievable group delay, which is the frequency derivative of the spectral phase, is limited. To limit the group delay required for compression, we use very short pieces of fiber (a few millimeters), pumped with rather short initial pulses. The short initial pulse duration and the use of a fiber with normal dispersion also lead to a relatively good spectral coherence of the supercontinuum.

We use $75 \%$ of the initial pulse energy as incident power on the microstructure fiber, while $25 \%$ is split off before the fiber for use in the cross-correlation SPIDER setup (see Section 2.C) used for characterization of the compressed pulses. An aspheric lens with a focal length of $4.5 \mathrm{~mm}$ is used to couple the pulses into the 5-mm-long microstructure fiber. The dispersion of the aspheric lens is precompensated with two double-chirped mirrors. Both ends of the fiber were cleaved at an angle of approximately $8^{\circ}$ in order to prevent backreflections into the laser. The output beam from the fiber was collimated with another lens with a short focal length $(10 \mathrm{~mm})$ and a thickness of $3.9 \mathrm{~mm}$, which contributes a significant amount of unwanted normal dispersion.

The microstructure fiber has a design often used for supercontinuum generation, containing a regular hexagonal array of air holes, with the central hole missing. Effectively, the central region is similar to a cylinder with a diameter of $2.6 \mu \mathrm{m}$. Figure 2 shows the measured dispersion profile (dotted curve) of this fiber along with supercontinuum spectra obtained with a pulse energy of $2.7 \mathrm{~nJ}$ launched into the fiber. Comparing the measured spectrum with the numerically simulated spectra (see Fig. 6 in Section 4) shows that both spectra span a bandwidth of more than $400 \mathrm{~nm}$ and qualitatively agree concerning their shapes, although they are slightly frequency shifted. The long wavelength tail from 950 to $1400 \mathrm{~nm}$ in the simulated spectra is suppressed in the measured one, because the spectral sensitivity of our CCD camera ranges from 180 to $1000 \mathrm{~nm}$.

Because of their importance for the supercontinuum coherence, we measured the power fluctuations of the transmitted pulses to be in the order of $3 \% \mathrm{rms}$ in a $50-\mathrm{kHz}$ bandwidth, while the rms fluctuations of the incident laser pulses were $\approx 4$ times smaller. We explain the larger output pulse fluctuations with the beam pointing fluctuations of the laser, which affect the input coupling efficiency.

We also performed some experiments with another fiber with a smaller core (1.7- $\mu \mathrm{m}$ diameter), which is slightly elliptical. Because the coupling efficiency into the fiber is strongly dependent on polarization, we placed the fiber in a rotational mount in order to adjust the fiber axes to the polarization of the input pulse. This fiber delivered broader spectra of more than $600-\mathrm{nm}$ width, but the shortest compressed pulses have been achieved with the other fiber.

The coupling efficiency into the fibers was estimated by comparison of the obtained spectra with numerically simulated spectra (see below) to be $\approx 20 \%$ for the fiber with the smaller core and $\approx 30 \%$ for the other one. Note that it is difficult to measure the launch efficiency for a short piece of fiber where the parts launched into the core and the cladding of the fiber cannot be easily separated.

\section{B. Pulse Shaper}

The pulse shaper setup (see Fig. 1) was designed for the broadest generated supercontinua, spanning a range from $\approx 400 \mathrm{~nm}$ to $1050 \mathrm{~nm}$. To obtain sufficient wavelength resolution (for sufficient group delay), we used a 640-pixel liquid crystal spatial light modulator (SLM), where the pixels are $97 \mu \mathrm{m}$ wide and are separated by $3-\mu \mathrm{m}$ gaps. The SLM was placed in the Fourier plane of a $4-f$ setup. ${ }^{19}$ For mapping the range of 400-1050 nm onto the SLM, we used gratings with 300 lines $/ \mathrm{mm}$ and spherical mirrors with a focal length of $300 \mathrm{~mm}$. To avoid excessive astigmatism, we used plane folding mirrors next to the gratings and operated the spherical mirrors with nearly normal incidence. The total power throughput of the pulse- 
shaping setup is only $15 \%$, because the gratings introduce considerable losses over such a large bandwidth.

The obtained compressor resolution is $\Delta \lambda \approx 1 \mathrm{~nm}$, corresponding to a maximum group delay $[\Delta \varphi / \Delta \omega$ $=\Delta \varphi /\left(2 \pi c \Delta \lambda / \lambda^{2}\right)$, where $\Delta \varphi$ is the phase difference between adjacent pixels] in the order of $330 \mathrm{fs}$ for $790-\mathrm{nm}$ light. It must be noted that the maximum group delay changes significantly from the short to the long wavelength side. The reason for this is that the spectrum is equally spaced in wavelength (after the grating), resulting in significant differences in the frequency bandwidth covered by each pixel. The result is that the maximum group delay for 400-nm and 1000-nm light is in the order of $85 \mathrm{fs}$ and $530 \mathrm{fs}$, respectively (assuming a maximum phase change of 1 rad between adjacent pixels).

\section{Cross-Correlation SPIDER}

After the pulse shaper, the pulses are fed into the pulsecharacterization setup. Because of the losses introduced in the continuum-generation process and in the pulseshaping apparatus, the average power of the pulses at the entrance of the pulse characterization setup is only $4 \mathrm{~mW}$. Owing to the huge bandwidth of the supercontinua, the spectral energy density is therefore rather low, making a conventional SPIDER ${ }^{20}$ measurement impossible. One would not be able to obtain a detectable SPIDER signal over the entire bandwidth of the spectrum. We therefore decided to build a cross-correlation SPIDER ${ }^{21}$ using the laser pulse for generating the strongly linearly chirped reference pulse and feeding the continuum into the "replica" arm. In principle the setup is the same as that described in Ref. 22 , except that we do not use a reflection from the glass block to generate the replicas but instead use the entire continuum beam. Owing to the enormous bandwidths we were especially careful concerning two issues: the bandwidth of the beamsplitters and the sumfrequency-generation crystal we used. We used customdesigned 400- $\mu \mathrm{m}$-thick ultrabroadband dielectric beamsplitters optimized for ultralow dispersion over a range spanning from 450 to $1000 \mathrm{~nm}$. For sumfrequency generation we chose a $30-\mu \mathrm{m}$-thick BBO crystal cut for type-II interaction. The use of this crystal for sum-frequency mixing of a broadband input pulse with the quasi-cw upconversion spectral slices in the extraordinary axis ensures a conversion efficiency over a large spectral range, with the half-maximum points reached at 530 and $1030 \mathrm{~nm}$.

While performing the SPIDER measurements with the broad supercontinuum generated with the 1.7- $\mu \mathrm{m}$-corediameter fiber, we noticed that strong scattered light from the shorter wavelength region of the fundamental spectrum corrupted the SPIDER signal in the spectrometer above $400 \mathrm{~nm}$. We first tried to cut the shorter wavelength region of the supercontinuum by inserting a knife edge in the Fourier plane of the pulse-shaping setup. However, the second diffraction order of the short wavelengths still overlapped in the Fourier plane with the long wavelengths, and its scattered light in the spectrometer was still too strong compared with the SPIDER signal. Therefore we had to use a color glass filter (1-mm Schott GG 495) to cut all spectral components below $495 \mathrm{~nm}$, although this meant introducing still more dispersion.

\section{Adaptive Compression}

The spectral phase reconstructed from the SPIDER measurement is used as feedback to iteratively compress the pulse. One iteration step includes the measurement of the spectral phase and the correction of the compressor setting through the subtraction of values proportional to the measured spectral phase from the control voltages corresponding to all wavelength components. Such iterations are repeated until the pulse no longer changes significantly. Typically, three iteration steps are required to obtain the shortest pulse.

\section{EXPERIMENTAL RESULTS}

In this section, we present our experimental results obtained with two different microstructure fibers, where the core diameters are 2.6 and $1.7 \mu \mathrm{m}$, respectively. In both cases, the propagation length was $5 \mathrm{~mm}$.

\section{A. Microstructure Fiber with 2.6- $\mu \mathrm{m}$ Core Diameter}

For the measurements presented in this subsection, we used a 2.6- $\mu \mathrm{m}$-core-diameter microstructure fiber from Crystal Fibre (Birkerød, Denmark) with a zero-dispersion wavelength of $940 \mathrm{~nm}$. With this fiber we were able to generate very bright and broadband spectra with excellent spatial characteristics. A typical spectrum, ranging from 510 to $940 \mathrm{~nm}$, is shown in Fig. 3. Owing to the fact that the spectrum is not octave spanning, we did not need to use a color glass filter to suppress the shorter wavelengths (see Subsection 2.C). With these spectra, we experienced no difficulties concerning the SPIDER measurements; the recorded interferogram showed a good fringe visibility, so a reliable reconstruction of the spectral phase of the pulses was possible. With this fiber we were able to demonstrate successful compression to a FWHM pulse duration of $5.5 \mathrm{fs}$ (see Fig. 4). Figure 3 also shows the spectral phase before compression (demagnified to fit into the diagram) and after compression. The obtained pulse energy after the pulse shaper was measured to be $0.2 \mathrm{~nJ}$. As can be seen in Fig. 3, the spectral phase is flat over the full spectrum, except for the outer parts, where the spectral energy density is too low for reliable reconstruction of the spectral phase. This explains why the pulses are slightly longer than the theoretical transform-

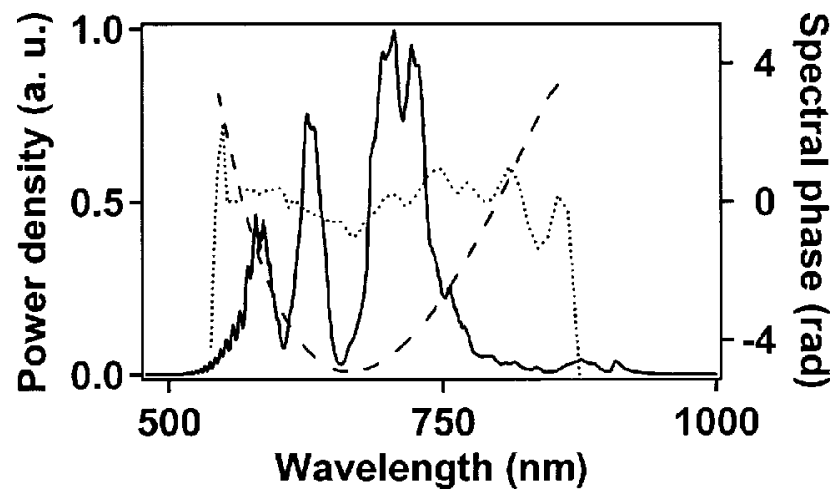

Fig. 3. Solid curve, experimentally measured output spectrum of the 2.6- $\mu \mathrm{m}$-core-diameter fiber; dashed curve, the reconstructed spectral phase of the generated pulses without compression, divided by 200; dotted curve, the spectral phase with compression (without rescaling). 


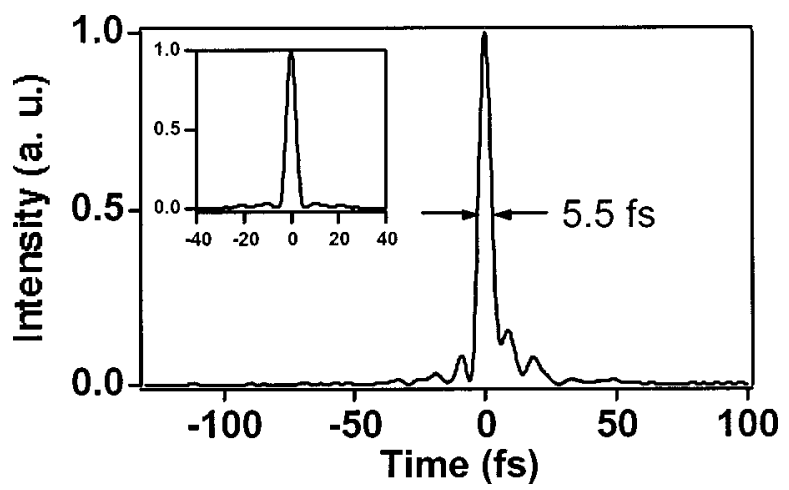

Fig. 4. Reconstructed temporal pulse profile showing a FWHM pulse duration of $5.5 \mathrm{fs}$; the inset shows the calculated transform-limited pulse shape.

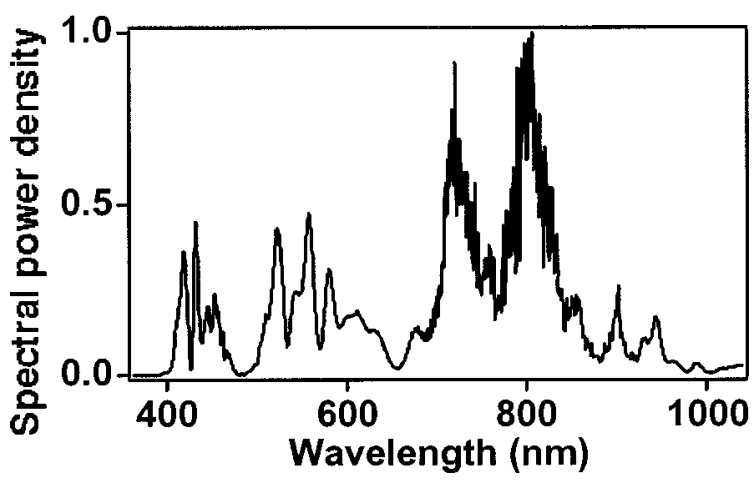

Fig. 5. Experimentally measured supercontinuum from the 1.7$\mu \mathrm{m}$-core-diameter fiber exhibiting strong modulations and interferences in the spectral region of the input laser pulse (700-900 $\mathrm{nm})$.

limited pulse duration of $4.8 \mathrm{fs}$ (see inset in Fig. 4) for the full spectrum or 5.3 fs for the spectrum between 545 and $870 \mathrm{~nm}$. (This spectral range corresponds to the bandwidth, over which the reconstruction of the spectral phase was possible.) To the best of our knowledge, these are the shortest pulses ever generated with a microstructure fiber.

\section{B. Microstructure Fiber with 1.7- $\mu \mathrm{m}$ Core Diameter}

For the generation of octave-spanning supercontinua, we used a polarization-maintaining microstructure fiber from Crystal Fibre with a core diameter of only $1.7 \mu \mathrm{m}$ and a zero-dispersion wavelength of $665 \mathrm{~nm}$. With this fiber we were able to produce spectra spanning from 400 to more than $1000 \mathrm{~nm}$. On the long-wavelength side the measurement was limited by the sensitivity of the silicon detector. A typical spectrum is shown in Fig. 5. The spectrum is strongly modulated and shows sharp spectral features in the wavelength range of the input laser beam. We believe these features to result from interferences between the core and the cladding modes, although it is not clear why this effect was observed only with the smaller core fiber.

We encountered several problems related to the characterization of these strongly structured broad supercontinua. As previously mentioned for the $2.6-\mu \mathrm{m}$ fiber, the spectral energy density in the wings of the spectrum was too low for the detection of an upconversion signal, even with the crosscorrelation SPIDER setup. With the much broader spectra from the $1.7-\mu \mathrm{m}$ core fiber, this problem was even more severe. Whereas the latter problem could possibly be overcome by use of pulses with higher energies, the mentioned sharp features on the spectra, caused by cladding modes, represent a more severe problem: They destroy the fringe visibility in the SPIDER interferogram and thus inhibit the correct reconstruction of the spectral phase. Note that although in principle one could remove the higher-order modes, e.g., using a mode cleaner (with focusing optics and pinhole), such operations are problematic when applied to these extremely broad spectra, which are very sensitive to effects like chromatic aberrations and additional dispersion. Additionally, the large difference in spot sizes for blue and near-infrared light limits the applicability of a mode cleaner for such huge bandwidths.

Even without interference from cladding modes, the spectra are strongly structured when the supercontinuum generation is optimized for large bandwidth. In particular, the spectral regions with nearly vanishing spectral intensity cause problems for the reconstruction of the phase in the SPIDER method.

Probably for these reasons, attempts to compress the broader spectra from the fiber with a smaller core were not successful. This shows that the best fiber for pulse compression is not necessarily the one generating the broadest spectra, and this holds true not only because of the issue of spectral coherence.

\section{THEORETICAL CONSIDERATIONS AND DISCUSSION OF RESULTS}

In this section, we investigate two important limiting factors for the dispersive compression of supercontinua: the limited spectral coherence and the effect of the limited compressor resolution. Probably the most fundamental limitation for supercontinuum compression is the limited coherence. The broadest spectra are often achieved when the fiber dispersion is anomalous in a large spectral range and the pulse intensity is high, as is possible with small mode areas. However, the launched pulses then correspond to solitons with a very high soliton order that subsequently break up into numerous weaker pulses. The latter can propagate partly as solitons and partly as dispersive radiation. The highly nonlinear nature of the involved processes can be extremely sensitive to the input pulse parameters. ${ }^{23-26}$ As a result, the generated output can vary significantly in spectral structure and spectral phase from pulse to pulse, even if the noise of the input pulses is at the quantum limit. With the large lowfrequency excess noise of typical mode-locked lasers, the coherence of the generated supercontinuum can become so poor that effective compression is not possible, because the spectral phase would have to be optimized separately for each single pulse.

However, the severity of the coherence issue depends strongly on a number of parameters, such as the fiber dispersion, the fiber length, and the initial pulse duration. In particular, the normal dispersion regime tends to lead to better coherence, although the achieved bandwidth is often smaller. ${ }^{8,10}$ Also, the coherence is favored by the 
use of short initial pulses. ${ }^{10,27,28}$ As a tentative explanation, the coherence can be poor for long pump pulses, because the Raman gain then occurs outside the initial pulse bandwidth and thus generates new spectral components by amplifying quantum noise. In contrast, for pump pulses with durations well below $100 \mathrm{fs}$, the Raman gain acts only on already-existing spectral components and therefore does not introduce that much noise. Finally, it has been found 29,30 that often the coherence is good below a certain threshold for the pulse energy, where a broad spectral width is already achieved, while the coherence rapidly degrades for higher pulse energies. Of course, this threshold depends on the fiber length.

Our experimental result, obtained in the normal dispersion regime with short initial pulses and a short fiber piece of only a 5-mm length, shows that compression down to $5.5 \mathrm{fs}$ is possible. In the following, we quantitatively investigate the expected coherence and other issues in the regime of our experiment. Note that calculations with the actual experimental parameters (or at least reasonable approximations) are required for the optimization of the setup and the interpretation of the obtained data. Conclusions from investigations in other parameter ranges usually cannot be used, since the behavior differs very much between different parameter regions.

We numerically simulated the propagation of pulses in the microstructure fibers using an algorithm that was described in Ref. 31. This algorithm models the effects of the nonlinear propagation, taking into account the effects of dispersion, self-phase modulation with self-steepening, four-wave mixing, and intrapulse Raman scattering. As already discussed in Subsection 2.A, the obtained spectra qualitatively agree with the experimentally obtained spectra. Exact quantitative agreement cannot be expected owing to uncertainties in the dispersion data (including possible variations along the fiber length) and the properties of the initial pulses, keeping in mind the sensitivity of the results on such parameters.

To estimate the spectral coherence, we performed the simulations for, e.g., 100 initial pulses that differ in energy (but not in shape) owing to some initial intensity noise with Gaussian probability distribution and an $\mathrm{rms}$ value of a few percent of the average intensity. Note that owing to the relatively long measurement times (e.g., several seconds for a SPIDER trace) the low-frequency classical noise is important, and this is far above the shotnoise level.

Figure 6 shows a simulation with 100 runs for 15-fs input pulses, a center wavelength of $790 \mathrm{~nm}$, an average power of $50 \mathrm{~mW}$ coupled into the fiber, and power fluctuations of $5 \% \mathrm{rms}$. The spectral amplitudes for all single runs as well as the average spectral amplitude are shown. In addition, the degree of coherence was calculated as in Ref. 10:

$$
\left|g_{12}^{(1)}(\lambda)\right|=\left|\frac{\left\langle E_{1}^{*}(\lambda) E_{2}(\lambda)\right\rangle}{\left[\left\langle\left|E_{1}(\lambda)\right|^{2}\right\rangle\left\langle\left|E_{2}(\lambda)\right|^{2}\right\rangle\right]^{1 / 2}}\right|,
$$

where the angle brackets denote an ensemble average over independently generated supercontinuum pairs $\left[E_{1}(\lambda), E_{2}(\lambda)\right]$. A value of at least 0.7 of the degree of coherence is a useful indicator of good compressibility.
For example, the peak power of the pulse is at least $70 \%$ of the maximum possible value for the given spectrum, if the coherence is at least 0.7 over the entire spectrum.

The coherence is found to be good in all spectral regions with significant spectral intensity (see Fig. 7). Note that the simulations can give a useful estimate for the coherence even if the spectral details are not all fully reproduced. Using these data, we simulated the pulse compression, where the compressor parameters were set according to the averaged spectral phase of the pulses, and the spectral intensity for wavelengths $>950 \mathrm{~nm}$ was

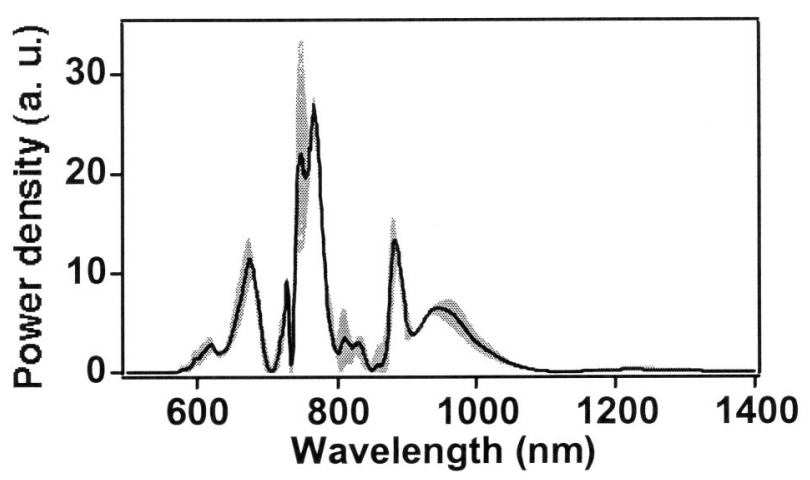

Fig. 6. Simulations for the 2.6- $\mu$ m-core-diameter fiber. Gray curves, 100 individual spectra; black solid curve, averaged spectrum.

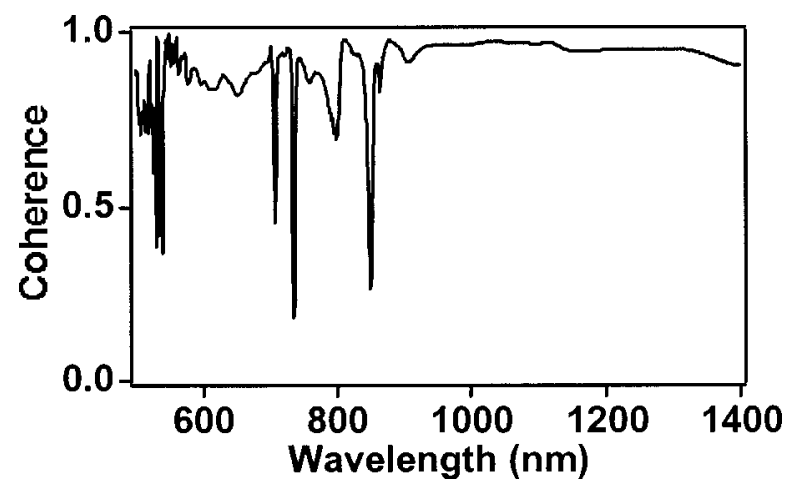

Fig. 7. Calculated degree of coherence for the 2.6- $\mu \mathrm{m}$-corediameter fiber for an ensemble of 100 pulses.

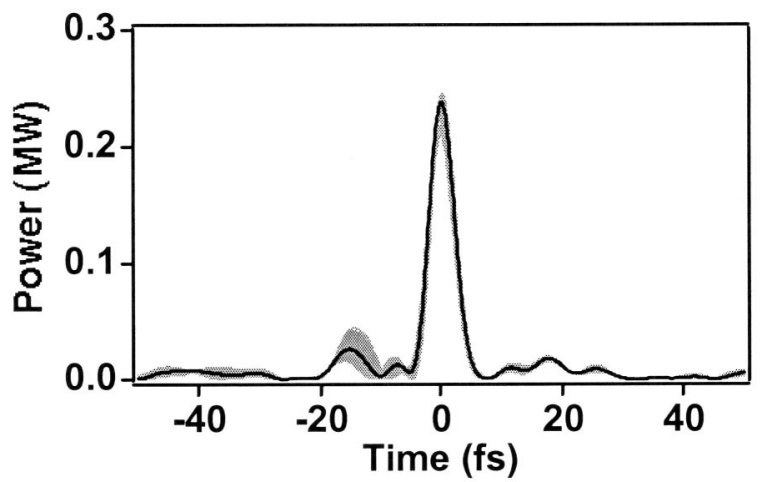

Fig. 8. Calculation of compressed pulses (2.6- $\mu \mathrm{m}$ fiber). Gray curves, 100 individual pulses compressed with the averaged spectral phase; black curve, average of the 100 compressed pulses. 


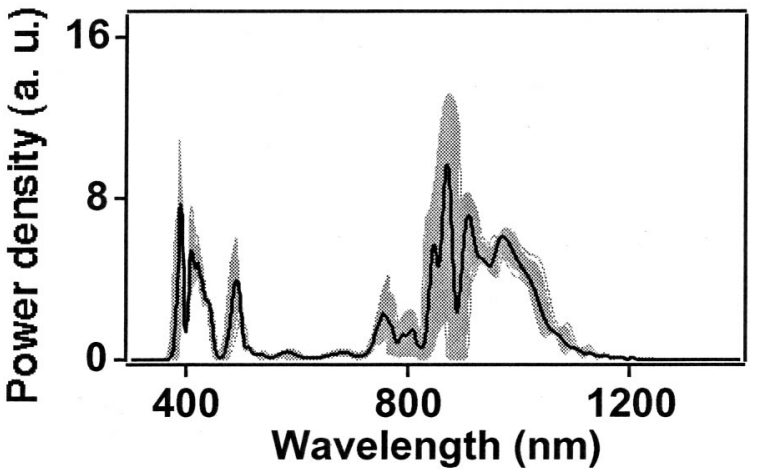

Fig. 9. Simulations for the 1.7- $\mu \mathrm{m}$-core-diameter fiber. Gray curves, 100 individual spectra; black solid curve, averaged spectrum.

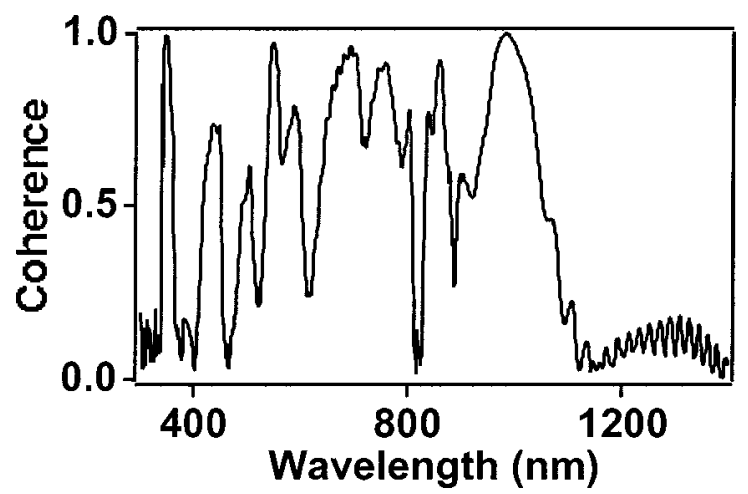

Fig. 10. Calculated degree of coherence for the 1.7- $\mu \mathrm{m}$-corediameter fiber for an ensemble of 100 pulses.

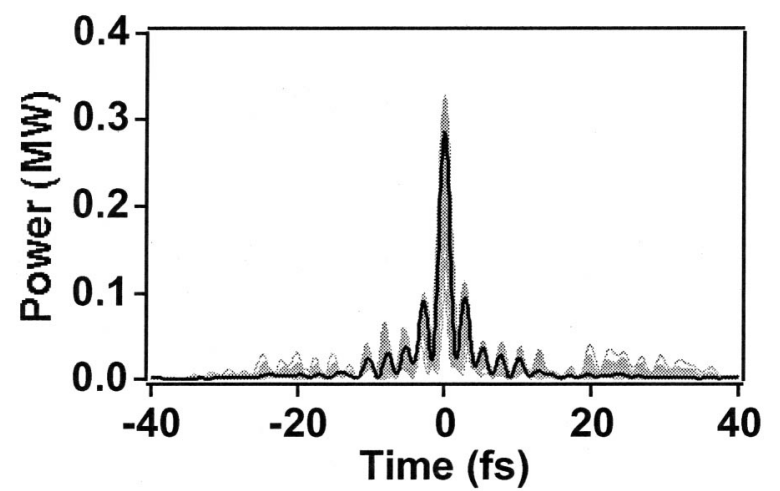

Fig. 11. Calculation of compressed pulses (1.7- $\mu \mathrm{m}$ fiber). Gray curves, 100 individual pulses compressed with the averaged spectral phase; black curve, average of the 100 compressed pulses.

suppressed as in the experiments. The spectral phase was corrected with only a finite compressor resolution similar to the resolution in the experiment. The simulation result demonstrated that the compression is not significantly affected by the limited coherence and the compressor resolution; the obtained FWHM pulse duration is on average 4.9 fs (see Fig. 8), close to the transform limit of $4.8 \mathrm{fs}$ for the averaged spectrum (cut at $950 \mathrm{~nm}$ ). This agrees with the successful experimental compression described in Subsection 3.A.
We did similar simulations for the fiber with a smaller core diameter of $1.7 \mu \mathrm{m}$, where the obtained spectra were significantly broader. Compared with the simulation result for the 2.6- $\mu \mathrm{m}$-core-diameter fiber, the spectral amplitudes show much stronger fluctuations for the same input power fluctuations (see Fig. 9). Here, the calculated coherence (Fig. 10) is significantly worse. The simulation of the compression (under the same conditions as above, except that only wavelengths $>1000 \mathrm{~nm}$ were suppressed as in the experiment) showed that although the average duration of the compressed pulses is still close to the transform limit, the amount of power in the temporal side lobes was significantly increased compared with the theoretical result for the compressed average pulse (see Fig. 11). Also we found that the limited compressor resolution has some degrading effect in this case. Despite these problems, the simulations suggest that in principle one may obtain compressed pulses with sub-2-fs average duration, although with only poor pulse quality. Even this was not achieved in the experiment, probably owing to the influence of cladding modes as explained in Subsection 3.B.

\section{CONCLUSIONS}

In conclusion, we have demonstrated pulse compression down to 5.5 fs from a supercontinuum generated in a microstructure fiber. The compression was achieved with iterative optimization of the setting of a liquid crystal SLM. The pulses were characterized by performing a cross-correlation SPIDER measurement.

To the best of our knowledge, the obtained pulse duration is the shortest demonstrated with this method. Key points were to use a rather short piece of fiber $(5 \mathrm{~mm})$, to start with short (15-fs) pump pulses, and to choose a fiber with normal dispersion, where the spectral coherence is better.

We have discussed various limitations of the general method and of the particular experimental results. For the obtained compression results, we could not use the full generated spectrum, because some parts did not have high enough spectral intensities for SPIDER characterization. For compression of broader spectra, more pulse energy would be needed. Even with higher pulse energies, the SPIDER characterization can be invalidated by strongly modulated spectral structures, particularly if spectral regions with low power density exist or if cladding modes destroy the fringe visibility. Finally, the spectral coherence tends to get worse for broader spectra, although this strongly depends on the chosen regime of fiber dispersion, initial pulse duration, etc. The coherence can greatly suffer from classical excess noise in the initial pulses, including their beam-pointing instability, which affects the launch efficiency. However, we have shown that, even with significant classical excess noise, the limited spectral coherence is not necessarily the most important limiting factor for pulse compression. If the coherence were the only limiting factor, we would expect compression to well below 5 fs to be feasible.

The authors thank John M. Dudley for useful discussions and the Swiss National Science Foundation for fi- 
nancial support. R. Paschotta's e-mail address is paschotta@phys.ethz.ch.

\section{REFERENCES}

1. P. Russell, "Photonic crystal fibers," Science 299, 358-362 (2003).

2. T. Südmeyer, F. Brunner, E. Innerhofer, R. Paschotta, K. Furusawa, J. C. Baggett, T. M. Monro, D. J. Richardson, and U. Keller, "Nonlinear femtosecond pulse compression at high average power levels using a large mode area holey fiber," Opt. Lett. 28, 1951-1953 (2003).

3. J. K. Ranka, R. S. Windeler, and A. J. Stentz, "Visible continuum generation in air-silica microstructure optical fibers with anomalous dispersion at $800 \mathrm{~nm}$," Opt. Lett. 25, 25-27 (2000).

4. I. Hartl, X. D. Li, C. Chudoba, R. K. Ghanta, T. H. Ko, J. G. Fujimoto, J. K. Ranka, and R. S. Windeler, "Ultrahighresolution optical coherence tomography using continuum generation in an air-silica microstructure optical fiber," Opt. Lett. 26, 608-610 (2001).

5. S. A. Diddams, D. J. Jones, J. Ye, S. T. Cundiff, J. L. Hall, J. K. Ranka, R. S. Windeler, R. Holzwarth, Th. Udem, and T. W. Hänsch, "Direct link between microwave and optical frequencies with a $300 \mathrm{THz}$ femtosecond laser comb," Phys. Rev. Lett. 84, 5102-5105 (2000).

6. R. Holzwarth, Th. Udem, T. W. Hänsch, J. C. Knight, W. J. Wadsworth, and P. St. J. Russel, "Optical frequency synthesizer for precision spectroscopy," Phys. Rev. Lett. 85, 22642267 (2000).

7. G. McConnell and E. Riis, "Ultra-short pulse compression using photonic crystal fibre," Appl. Phys. B: Photophys. Laser Chem. 78, 557-563 (2004).

8. S. Lako, J. Seres, P. Apai, J. Balazs, R. S. Windeler, and R. Szipöcs, "Pulse compression of nanojoule pulses in the visible using microstructure optical fiber and dispersion compensation," Appl. Phys. B: Photophys. Laser Chem. 76, 267-275 (2003).

9. M. Adachi, K. Yamane, R. Morita, and M. Yamashita, "Microstructure fiber feedback pulse compression," in Conference on Ultrafast Phenomena (Optical Society of America, Washington, D.C. 2004), poster ME47.

10. J. M. Dudley and S. Coen, "Coherence properties of supercontinuum spectra generated in photonic crystal and tapered optical fibers," Opt. Lett. 27, 1180-1182 (2002).

11. L. Gallmann, D. H. Sutter, N. Matuschek, G. Steinmeyer, and U. Keller, "Techniques for the characterization of sub10-fs optical pulses: a comparison," Appl. Phys. B: Photophys. Laser Chem. 70, S67-S75 (2000).

12. M. Nisoli, S. De Silvestri, and O. Svelto, "Generation of high energy $10 \mathrm{fs}$ pulses by a new pulse compression technique," Appl. Phys. Lett. 68, 2793-2795 (1996).

13. W. Kornelis, J. Biegert, J. W. G. Tisch, M. Nisoli, G. Sansone, C. Vozzi, S. De Silvestri, and U. Keller, "Single-shot kilohertz characterization of ultrashort pulses by spectral phase interferometry for direct electric-field reconstruction," Opt. Lett. 28, 281-283 (2003).

14. B. Schenkel, J. Biegert, U. Keller, C. Vozzi, M. Nisoli, G. Sansone, S. Stagira, S. De Silvestri, and O. Svelto, "Generation of 3.8-fs pulses from adaptive compression of a cascaded hollow fiber supercontinuum," Opt. Lett. 28, 19871989 (2003).

15. K. Yamane, Z. Zhang, K. Oka, R. Morita, and M. Yamashita, "Optical pulse compression to $3.4 \mathrm{fs}$ in the monocycle region by feedback phase compensation," Opt. Lett. 28, 2258-2261 (2004).

16. K. Yamane, T. Kito, R. Morita, and M. Yamashita, "2.8-fs transform-limited optical-pulse generation and characterization," in Conference on Ultrafast Phenomena (Optical Society of America, Washington, D.C., 2004), poster ThD16.

17. N. Matuschek, F. X. Kärtner, and U. Keller, "Theory of double-chirped mirrors," IEEE J. Sel. Top. Quantum Electron. 4, 197-207 (1998).

18. R. L. Fork, O. E. Martinez, and J. P. Gordon, "Negative dispersion using pairs of prisms," Opt. Lett. 9, 150-152 (1984).

19. A. M. Weiner, "Femtosecond pulse shaping using spatial light modulators," Rev. Sci. Instrum. 71, 1929-1960 (2000).

20. C. Iaconis and I. A. Walmsley, "Spectral phase interferometry for direct electric-field reconstruction of ultrashort optical pulses," Opt. Lett. 23, 792-794 (1998).

21. M. Zavelani-Rossi, G. Cerullo, S. De Silvestri, L. Gallmann, N. Matuschek, G. Steinmeyer, U. Keller, G. Angelow, V. Scheuer, and T. Tschudi, "Pulse compression over a 170$\mathrm{THz}$ bandwidth in the visible by use of only chirped mirrors," Opt. Lett. 26, 1155-1157 (2001).

22. L. Gallmann, D. H. Sutter, N. Matuschek, G. Steinmeyer, U. Keller, C. Iaconis, and I. A. Walmsley, "Characterization of sub-6-fs optical pulses with spectral phase interferometry for direct electric-field reconstruction," Opt. Lett. 24, 1314-1316 (1999).

23. A. L. Gaeta, "Nonlinear propagation and continuum generation in microstructured optical fibers," Opt. Lett. 27, 924-926 (2002).

24. A. V. Husakou and J. Hermann, "Supercontinuum Generation of Higher-Order Solitons by Fission in Photonic Crystal Fibers," Phys. Rev. Lett. 87, 203901 (2001).

25. J. M. Dudley, X. Gu, L. Xu, M. Kimmel, E. Zeek, P. O’Shea, R. Trebino, S. Coen, and R. S. Windeler, "Cross-correlation frequency resolved optical gating analysis of broadband continuum generation in photonic crystal fiber: simulations and experiments," Opt. Express 10, 1215-1221 (2002), http://www.opticsexpress.org.

26. X. Gu, L. Xu, M. Kimmel, E. Zeek, P. O'Shea, A. P. Shreenath, R. Trebino, and R. S. Windeler, "Frequencyresolved optical gating and single-shot spectral measurements reveal fine structure in microstructure-fiber continuum," Opt. Lett. 27, 1174-1176 (2002).

27. K. L. Corwin, N. R. Newbury, J. M. Dudley, S. Coen, S. A. Diddams, K. Weber, and R. S. Windeler, "Fundamental noise limitations to supercontinuum generation in microstructure fiber," Phys. Rev. Lett. 90, 113904 (2003).

28. K. L. Corwin, N. R. Newbury, J. M. Dudley, S. Coen, S. A. Diddams, B. R. Washburn, K. Weber, and R. S. Windeler, "Fundamental amplitude noise limitations to supercontinuum spectra generated in a microstructured fiber," Appl. Phys. Lett. 77, 269-277 (2003).

29. G. Chang, T. B. Norris, and H. Winful, "Optimization of supercontinuum generation in photonic crystal fibers for pulse compression," Opt. Lett. 28, 546-548 (2003).

30. J. M. Dudley and S. Coen, "Fundamental limits to few-cycle pulse generation from compression of supercontinuum spectra generated in photonic crystal fiber," Opt. Express 12, 2423-2428 (2004), http://www.opticsexpress.org.

31. J. H. V. Price, T. M. Monro, K. Furusawa, W. Belardi, J. C. Baggett, S. Coyle, C. Netti, J. J. Baumberg, R. Paschotta, and D. J. Richardson, "UV generation in a pure silica holey fiber,” Appl. Phys. B: Photophys. Laser Chem. 77, 291-295 (2003). 